\title{
Ewing's sarcoma and primitive neuroectodermal tumour (ES/PNET) presenting as a breast mass
}

\author{
SUEBWONG CHUTHAPISITH ${ }^{1}$, WILAIRAT PRASERT ${ }^{1}$, MALEE WARNNISSORN ${ }^{2}$, \\ KANAPON PRADNIWAT ${ }^{2}$, VICHIEN SRIMUNINNIMIT ${ }^{3}$ and TAMNIT ANGSUSINHA ${ }^{4}$ \\ Departments of ${ }^{1}$ Surgery, ${ }^{2}$ Pathology and ${ }^{3}$ Medicine; ${ }^{4}$ Thanyarak Breast Imaging Center, \\ Faculty of Medicine Siriraj Hospital, Mahidol University, Bangkok 10700, Thailand
}

Received February 9, 2012; Accepted April 24, 2012

DOI: $10.3892 / 01.2012 .698$

\begin{abstract}
Ewing's sarcoma/primitive neuroectodermal tumour (ES/PNET) is a rare tumour usually detected in young individuals and uncommonly found within the breast tissue. In this case report, we examined a 46-year-old patient, who developed a lump on her breast and was later diagnosed with ES/PNET. Clinical presentation, age at development and radiological findings were of interest and were discussed. Diagnosis of the tumour was confirmed using various immunohistochemical studies and the presence of a translocation, $t(11 ; 22)$. A literature review of this rare condition was also included.
\end{abstract}

\section{Introduction}

Ewing's sarcoma/primitive neuroectodermal tumour (ES/ PNET) is a member of the Ewing's sarcoma family of tumours (ESFT). Extraskeletal ES is a manifestation of ES within the soft tissues, and an ES tumour presenting as a breast mass is unusual. ES typically occurs in adolescents and young adults aged between 10 and 20 years (1). The diagnosis of ES/PNET requires panels of immunohistochemical study and the presence of a $\mathrm{t}(11 ; 22)$ translocation detected through fluorescent in situ hybridization (FISH). This case report examines a rare case of ES/PNET observed in a 46-year-old woman, who developed a painless and progressive tumour presenting as a breast mass. The patient's family provided their consent to the study.

Correspondence to: Dr Suebwong Chuthapisith, Division of Head, Neck and Breast Surgery, Department of Surgery, Faculty of Medicine Siriraj Hospital, Mahidol University, Bangkok 10700, Thailand

E-mail: sisuebwong@mahidol.ac.th; suebwong.chu@mahidol.ac.th

Key words: breast, Ewing's sarcoma/primitive neuroectodermal tumour, extraskeletal

\section{Case report}

In 2010, a 46-year-old woman presented a painless and progressive mass in her right breast for 1 month. Upon diagnostic mammography and ultrasonography, a suspicious mass of $4 \mathrm{~cm}$ in size was identified (Fig. 1A and B). Following this, a biopsy was performed and the initial diagnosis was a neuroendocrine carcinoma, as the tumour was positive for synaptophysin, but negative for ER, PR, HER2/neu and CK8/18.

Following a core needle biopsy, the tumour increased rapidly in size and became $12 \times 12 \mathrm{~cm}$ within 1 month. The patient received chemotherapy containing cyclophosphamide, adriamycin and vincristine for 6 cycles, to which the tumour responded well and shrank to $1.5 \times 2.5 \mathrm{~cm}$. Whole breast radiation was continued at a dose of $50 \mathrm{~Gy}$; however, following completion, the tumour progressed quickly to $6 \times 7 \mathrm{~cm}$ within 1 month. A CT scan of the chest revealed a $0.6 \mathrm{~cm}$ sized nodule in the right lung. Therefore, platinum and etoposide were commenced for 3 cycles, followed by paclitaxel and carboplatin. Despite this treatment, the tumour did not respond to the chemotherapy administered and the disease progressed; the patient was then referred to our institute.

While the patient was in our institute, the tumour invaded through the skin and grew to $20 \times 15 \mathrm{~cm}$. A review of a previous biopsy demonstrated ES/PNET as a malignant small round cell neoplasm (Fig. 2A). Immunohistochemical staining was performed for vimentin (clone V9, dilution 1:500), CD20 (clone L26, dilution 1:2,000), CD99 (clone 12E7, ready-to-use), desmin (clone D33, dilution 1:1,000), S-100 (polyclonal, dilution 1:10,000) from DakoCytomation (Glostrup, Denmark); FLI-1 (clone MRQ-1, ready-to-use), CD56 (clone 123C3.D5, ready-to-use), AE1/AE3 (readyto-use), chromogranin A (LK2H10, ready-to-use), CD30 (clone Ber-H2, ready-to-use) from Cell Marque (Rocklin, CA, USA); CK8/18 (clone 5D3, dilution 1:300) from NEOMARKERS (Fremont, CA, USA); synaptophysin (clone SP11, dilution 1:500) from Diagnostic Biosystems (Pleasanton, CA, USA); CD45 (clone PD7/26/16\&2B11, dilution 1:2,000) from BioGenex (Fremont, CA, USA); CD3 (clone LN10, ready-to-use), TdT (clone NPT26, ready-touse), TTF-1 (clone SPT24, ready-to-use) from Novocastra (Newcastle, UK) and Pax-5 (clone SP34, ready-to-use) 

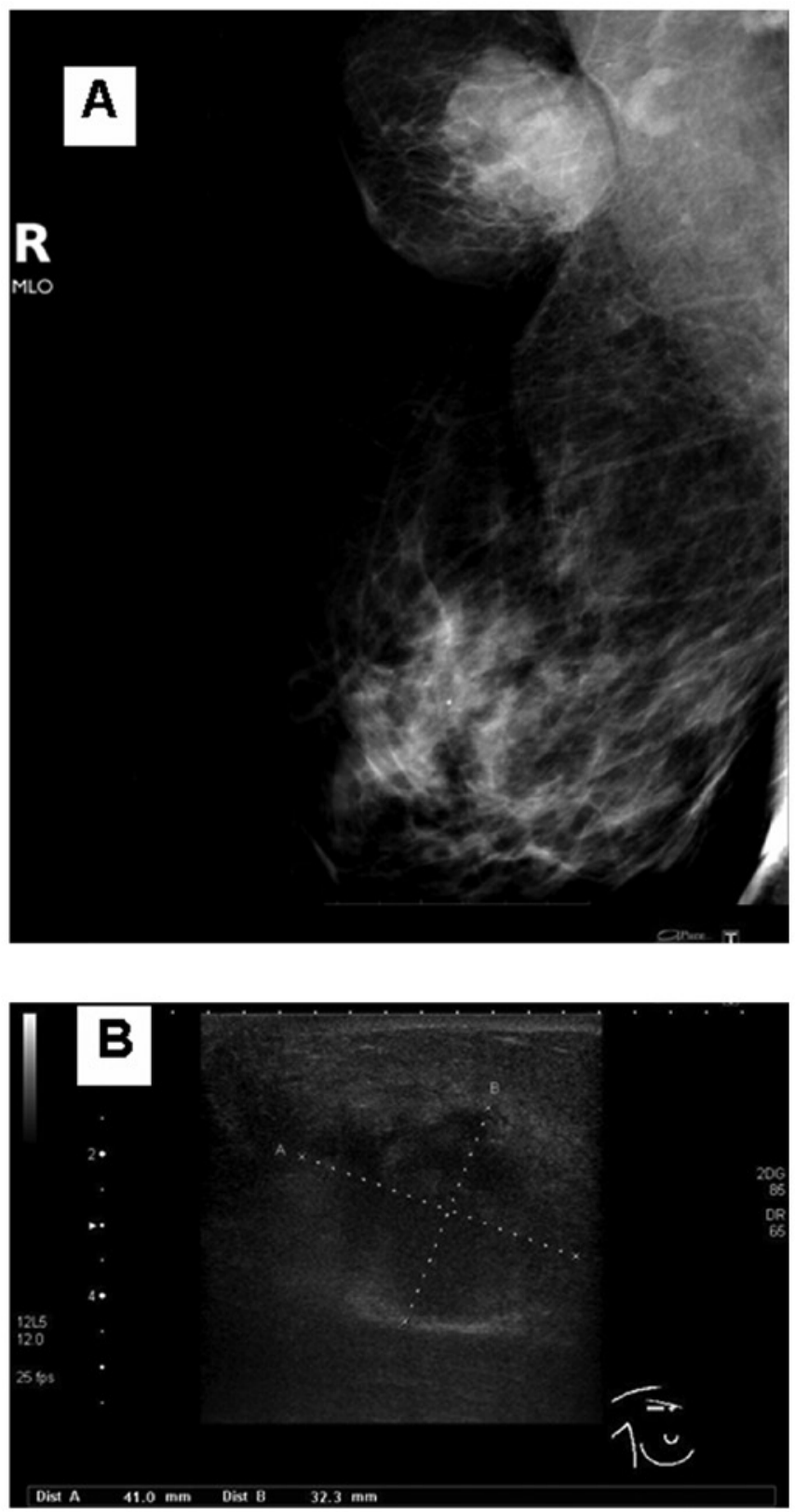

Figure 1. (A) A mediolateral oblique (MLO) view and (B) ultrasonography of the right breast at first presentation in 2010. A circumscribed mass was identified in the upper breast. At the deep margin, the mass attached to the soft tissue underneath.

from Ventana (Tucson, AZ, USA). An automated immunostainer, BenchMark XT (Ventana), with a polymer-based DakoEnVision detection system (DakoCytomation), were used. The tumour cells marked with vimentin, FLI-1 (diffuse nuclear staining), CD99 (membrane staining; intense) and CD56 were negative for desmin, AE1/AE3, CK8/18, chromogranin A, synaptophysin, S100, CD45, CD3, CD20, PAX5, CD30, TdT and TTF-1 (Fig. 2B and C). These results were consistent with ES/PNET. Following this, two cycles of paclitaxel and carboplatin were initiated. Progression of the disease was observed and a modified radical mastectomy with local skin flap coverage was performed (Fig. 3A and B). The tumour was localized in the upper outer part of the right breast, with involvement of the underlying skeletal muscle,

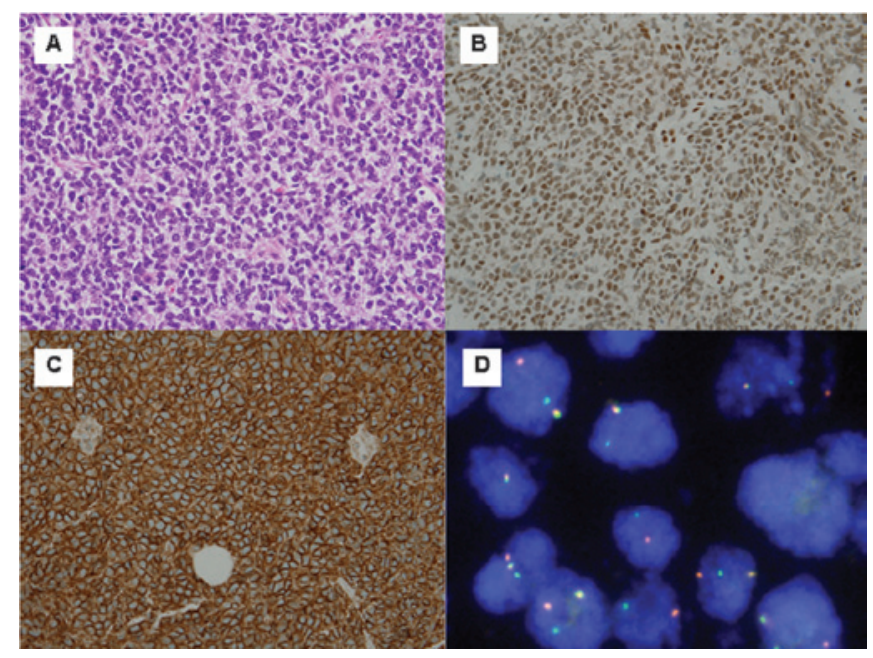

Figure 2. (A) Ewing's sarcoma/primitive neuroectodermal tumour (ES/ PNET) as a small round cell neoplasm, exhibits relatively uniform ovalshaped nuclei, finely dispersed chromatin and pale eosinophilic to clear cytoplasms. The cytoplasmic borders were indistinct (H\&E staining; magnification, $\mathrm{x} 400$ ). (B) FLI-1 immunoreactivity in ES cell nuclei (magnification, $\mathrm{x} 400$ ). (C) Positivity for CD99 on cell membranes (magnification, $\mathrm{x} 400$ ). (D) Fluorescence in situ hybridization with the LSI EWSR1 (22q12) Break Apart Probe demonstrated chromosomal rearrangement in the EWSRI gene region. Cells with $\mathrm{t}(22 \mathrm{q} 12)$ revealed one fusion, one orange and one green signal pattern.

measuring $20.5 \times 15.5 \times 12 \mathrm{~cm}$. The surgical margins were free from the tumour, and lymphovascular invasion and metastatasis to the axillary lymph nodes ( 3 of 24 nodes), were reported. An additional FISH study confirmed the presence of EWSRl gene translocation (Fig. 2D). Thus, ES/PNET was the final diagnosis.

Only 1 month following the surgery, the patient developed recurrent disease on the chest wall, as well as multiple lung nodules. The disease progressed rapidly and two months following surgery, the patient succumbed to respiratory failure due to pulmonary metastasis.

\section{Discussion}

We report a rare case of extraskeletal ES/PNET, presenting with a rapidly growing palpable breast lump. Age at presentation, radiological findings and immunohistochemical findings were of interest and documented in this report.

ES and PNET are typically undifferentiated $(1,2)$. Translocation $\mathrm{t}(11 ; 22)(\mathrm{q} 24 ; \mathrm{q} 12)$ resulting in $E W S / F L I 1$ fusion, which can be identified in more than $90 \%$ of ES/PNET cases, is the genetic hallmark of ES/PNET (3). In cases with classic morphology where other small round cell neoplasms have been excluded immunohistochemically, the expression of CD99 cell surface antigen (product of the $M I C-2$ gene) is required to support ES/PNET diagnosis (4,5).

The majority of patients with ES/PNET are 10-20 years old (1), and other small studies of adult ES/PNET from the Royal Marsden, the Memorial Sloan-Kettering and the Dana-Faber Cancer Centers have reported a median age of 24-27 years $(1,6,7)$. However, our patient was 46 years of age at the time of diagnosis, which is unusual. 


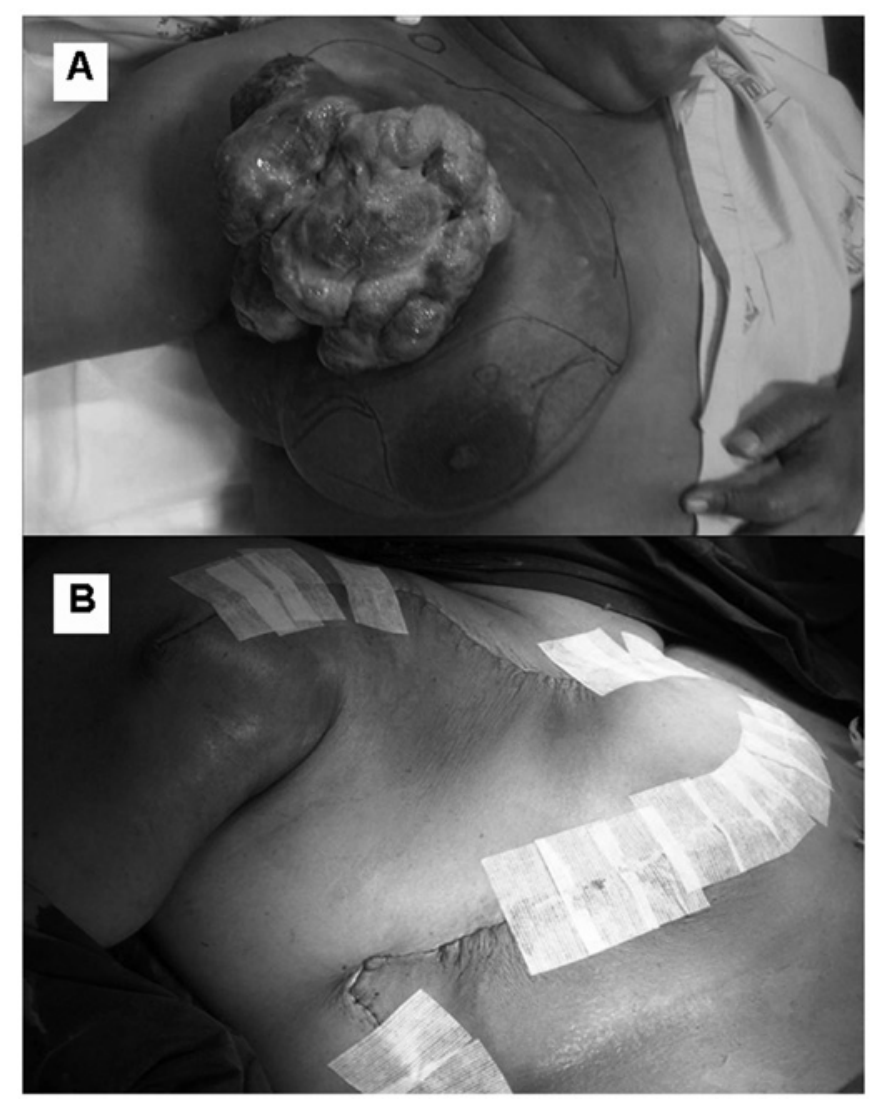

Figure 3. (A) Tumor prior to the operation in 2011, positioned in the upper outer part of the breast, invaded into the skin and grew to $20 \times 15 \mathrm{~cm}$. (B) Local skin flap coverage following modified radical mastectomy.

ES/PNET development within breast tissue was unlikely to be diagnosed upon first presentation. Findings from mammography and ultrasonography breast images may vary as they could be from a hypoechoic mass with posterior enhancement or heterogeneous mass with a necrotic area $(8,9)$. In this patient, a well-circumscribed mass was documented (Fig. 1A and B), which possibly arose from the soft tissue beneath the breast. Precise identification of the tumour location (chest wall soft tissue) and recognition of variation in radiographic findings described in this tumour may be a clue for radiologists to avoid misinterpreting the tumour as a breast mass that might preclude the diagnosis of soft tissue tumours.

Diagnosis of ES/PNET in this patient was based on the immunohistochemical staining results, which documented a positive expression of CD56, CD99, FLI-1, synaptophysin and vimentin, but a negative expression of AE1/AE3, CK8/18, EMA, chromogranin A, CD45 (LCA), HER-2, ER and PR. An additional FISH study confirmed the presence of EWSR1 gene translocation. The positive expression of CD99 (MIC2), a cell surface glycoprotein involved in cell adhesion, plays a crucial role in the diagnosis of ES/PNET (10). However, CD99 may also be expressed in other tumours, including metaplastic carcinoma of the breast, neuroendocrine carcinoma, lymphoma and rhabdomyosarcoma (11). In our patient, small cell carcinoma, neuroendocrine carcinoma and malignant lymphoma were excluded by negative staining for cytokeratins, chromogranin A and LCA.

$\mathrm{ES} / \mathrm{PNET}$ is an aggressive tumour with a high incidence of local recurrence and distant metastasis. A combination of multiple modalities, including surgery, chemotherapy and radiation therapy, was the most appropriate treatment for our patient (1). All members of the ESFT tend to share the propensity for metastatic spread. Consistent use of systemic chemotherapy to treat localised ESFT effectively improved the 5-year survival rate from 5 to $10 \%$ up to $65 \%$, which is primarily due to the elimination of micrometastases (12-14). Although the optimum combination chemotherapy has not yet been established, a regimen containing vincristine, adriamycin, cyclophosphamide and actinomycin D, was the standard first-line treatment for patients with localized disease (12). In patients with unresectable or metastatic disease, palliative chemotherapy may be useful. In this patient, vincristine, adriamycin and cyclophosphamide were utilized at the first instance. The tumour appeared to respond well initially, but became resistant to various chemotherapeutic agents, which resulted in progression of the disease.

The role of radiation therapy in the treatment of ES/PNET is unclear. However, the use of radiation therapy combined with surgery, in order to control local disease, is proving to be helpful (1). Our patient did not respond to radiation therapy treatment and the tumour grew rapidly following cessation of radiation. This is similar to a previous study where a $78 \%$ disease progression rate was observed in 14 patients treated with radiotherapy plus chemotherapy (15).

In conclusion, we report a rare case of ES/PNET presenting as a breast mass. Clinical presentation mimicked invasive breast cancer. The tumour did not respond well to multimodality treatment and local and distant metastasis occurred less than 2 years after first diagnosis.

\section{References}

1. Baldini EH, Demetri GD, Fletcher CD, Foran J, Marcus KC and Singer S: Adults with Ewing's sarcoma/primitive neuroecto-dermal tumor: adverse effect of older age and primary extraosseous disease on outcome. Ann Surg 230: 79-86, 1999.

2. Dehner LP: Primitive neuroectodermal tumor and Ewing's sarcoma. Am J Surg Pathol 17: 1-13, 1993.

3. Jambhekar NA, Bagwan IN, Ghule P, Shet TM, Chinoy RF, Agarwal S, et al: Comparative analysis of routine histology, immunohistochemistry, reverse transcriptase polymerase chain reaction, and fluorescence in situ hybridization in diagnosis of Ewing family of tumors. Arch Pathol Lab Med 130: 1813-1818, 2006.

4. De Alava E and Gerald WL: Molecular biology of the Ewing's sarcoma/primitive neuroectodermal tumor family. J Clin Oncol 18: 204-213, 2000.

5. Turc-Carel C, Philip I, Berger MP, Philip T and Lenoir GM: Chromosome study of Ewing's sarcoma (ES) cell lines. Consistency of a reciprocal translocation $\mathrm{t}(11 ; 22)(\mathrm{q} 24 ; \mathrm{q} 12)$. Cancer Genet Cytogenet 12: 1-19, 1984.

6. Martin RC and Brennan MF: Adult soft tissue Ewing sarcoma or primitive neuroectodermal tumors: predictors of survival? Arch Surg 138: 281-285, 2003.

7. Verrill MW, Judson IR, Harmer CL, Fisher C, Thomas JM and Wiltshaw E: Ewing's sarcoma and primitive neuroectodermal tumor in adults: are they different from Ewing's sarcoma and primitive neuroectodermal tumor in children? J Clin Oncol 15: 2611-2621, 1997.

8. Da Silva BB, Lopes-Costa PV, Pires CG, Borges RS and da Silva RG Jr: Primitive neuroectodermal tumor of the breast. Eur J Obstet Gynecol Reprod Biol 137: 248-249, 2008. 
9. Maxwell RW, Ghate SV, Bentley RC and Soo MS: Primary primitive neuroectodermal tumor of the breast. J Ultrasound Med 25: 1331-1333, 2006.

10. Tamura G, Sasou S, Kudoh S, Kikuchi J, Ishikawa A, Tsuchiya T, et al: Primitive neuroectodermal tumor of the breast: immunohistochemistry and fluorescence in situ hybridization. Pathol Int 57: 509-512, 2007.

11. Milanezi F, Pereira EM, Ferreira FV, Leitao D and Schmitt FC: CD99/MIC-2 surface protein expression in breast carcinomas. Histopathology 39: 578-583, 2001.

12. Paulussen M, Ahrens S, Dunst J, Winkelmann W, Exner GU, Kotz R, et al: Localized Ewing tumor of bone: final results of the cooperative Ewing's Sarcoma Study CESS 86. J Clin Oncol 19: $1818-1829,2001$
13. Rosito P, Mancini AF, Rondelli R, Abate ME, Pession A, Bedei L, et al: Italian Cooperative Study for the treatment of children and young adults with localized Ewing sarcoma of bone: a preliminary report of 6 years of experience. Cancer 86: 421-428, 1999.

14. Verrill MW, Judson IR, Wiltshaw E, Thomas JM, Harmer CL and Fisher C: The use of paediatric chemotherapy protocols at full dose is both a rational and feasible treatment strategy in adults with Ewing's family tumours. Ann Oncol 8: 1099-1105, 1997.

15. Kushner BH, Hajdu SI, Gulati SC, Erlandson RA, Exelby PR and Lieberman PH: Extracranial primitive neuroectodermal tumors. The Memorial Sloan-Kettering Cancer Center experience. Cancer 67: 1825-1829, 1991. 